\title{
Confinement Properties in the Multi-Instanton System
}

\author{
M. Fukushima $^{\text {a* }}{ }^{\text {, H. Suganuma }}{ }^{\text {a }}$, A. Tanaka ${ }^{a}$, H. Toki ${ }^{a}$ and S. Sasaki ${ }^{b}$ \\ ${ }^{a}$ Research Center for Nuclear Physics (RCNP), Osaka University, Ibaraki, Osaka 567, Japan \\ bYukawa Institute for Theoretical Physics, Kyoto University, Kyoto 606-01, Japan
}

\begin{abstract}
We investigate the confinement properties in the multi-instanton system, where the size distribution is assumed to be $\rho^{-5}$ for the large instanton size $\rho$. We find that the instanton vacuum gives the area law behavior of the Wilson loop, which indicates existence of the linear confining potential. In the multi-instanton system, the string tension increases monotonously with the instanton density, and takes the standard value $\sigma \simeq 1 \mathrm{GeV} / \mathrm{fm}$ for the density $(N / V)^{\frac{1}{4}}=200 \mathrm{MeV}$. Thus, instantons directly relate to color confinement properties.
\end{abstract}

\section{Topological Objects in QCD Vacuum}

In the QCD vacuum, there are two non-trivial topological objects, instantons and monopoles, which belong different sectors of physics. It is believed that these objects are independently important for understanding the non-perturbative properties. An instanton appears as a classical and non-trivial solution in the Yang-Mills theory corresponding to the homotopy group, $\pi_{3}\left(S U\left(N_{c}\right)\right)=Z_{\infty}$ [1]. Instantons are important for the phenomena related to the $U_{A}(1)$ anomaly and chiral symmetry breaking [2, 3]. On the other hand, QCD is reduced to an abelian gauge theory with QCD-monopoles after performing the abelian gauge fixing 何. QCDmonopoles appear as the topological defects corresponding to the nontrivial homotopy group $\pi_{2}\left(S U\left(N_{c}\right) / U(1)^{N_{c}-1}\right)=Z_{\infty}^{N_{c}-1}$. Condensation of monopoles plays an essential role on color confinement and chiral symmetry breaking [ [5]-[7].

Until now, there has been no evidence that the instanton has anything to do with color confinement. However, recent studies [7]-[16] suggest that instantons directly relate to monopoles, whose condensation provides an interpretation of the confinement mechanism. In this paper, we study the further relation between instantons and the confinement properties.

\footnotetext{
*e-mail: masa@rcnp.osaka-u.ac.jp
}

\section{Correlation between Instantons and Monopoles}

Recently, it has been found that there exists a strong correlation between instantons and monopoles in the abelian projected theory of QCD, both in the analytical and lattice frameworks [7]-14. In the maximally abelian gauge, a monopole trajectory seems to be localized around the center of an instanton [12,13]. We studied the multi-instanton system in terms of monopole condensation numerically [15,16]. When the instanton density is high and instantons overlap each other, there appears an very long and highly complicated monopole trajectory. This monopole trajectory covers the entire physical volume $\mathbf{R}^{4}$ in the multi-instanton system. [15,16]. In the lattice QCD simulation, the large monopole clustering is observed in the confinement phase, which includes many instantons and anti-instantons as the topological excitation [14. Thus, such long and complicated monopole trajectory is a signal of monopole condensation, which is responsible for color confinement.

Therefore, instantons would play a relevant role on the confinement mechanism via the promotion of monopole condensation. In order to clarify the relation of instantons with color confinement, we study the multi-instanton system, which is regarded to hold the essence of the non- 
perturbative QCD vacuum.

\section{Multi-Instanton Configuration}

The gauge configuration of an instanton with the size $\rho$ and the center $z$ in the singular gauge is expressed as

$A_{\mu}^{I}(x ; z, \rho, O)=\frac{i \tau^{a} \rho^{2} O^{a b} \bar{\eta}_{\mu \nu}^{b}(x-z)_{\nu}}{(x-z)^{2}\left[(x-z)^{2}+\rho^{2}\right]}$,

where $O^{a b}$ denotes the $\mathrm{SU}(2)$ color orientation matrix and $\bar{\eta}_{\mu \nu}^{a}$ the 't Hooft symbol. The antiinstantons configuration $A_{\mu}^{\bar{I}}$ is obtained by replacing $\bar{\eta}_{\mu \nu}^{a}$ with $\eta_{\mu \nu}^{a}$.

The multi-instanton ensemble is characterized by the size distribution, the randomness of the color orientation and the density of instantons [15,16. 16. The size distribution has to follow the one loop result, $f(\rho) \sim \rho^{b-5}$ with $b=11 N_{c} / 3$, in the ultra-violet region. For the large size, the ordinary instanton liquid models suggest that the size distribution behaves as $f(\rho) \sim 1 / \rho^{5}$ due to the infrared repulsive force [3]. Therefore, we assume the size distribution as

$f(\rho)=\frac{1}{\left(\frac{\rho}{\rho_{1}}\right)^{\nu}+\left(\frac{\rho_{2}}{\rho}\right)^{b-5}}$

with size parameters $\rho_{1}$ and $\rho_{2}$, which should satisfy the normalization condition, $\int_{0}^{\infty} d \rho f(\rho)=1$. The maximum of the distribution is fixed to the standard probable size $\rho_{0}=0.4 \mathrm{fm}$. In this calculation, we take $\nu=5$ for the behavior of the size distribution on large instantons, although we are planning to investigate how the long-range physics depend on the infrared behavior of the size distribution.

The multi-instanton configurations are approximated as the sum of instanton and anti-instanton solutions,

$$
\begin{aligned}
A_{\mu}(x)=\sum_{k} A_{\mu}^{I}\left(x ; z_{k}, \rho_{k}, O_{k}\right) & \\
& +\sum_{\bar{k}} A_{\mu}^{\bar{I}}\left(x ; z_{\bar{k}}, \rho_{\bar{k}}, O_{\bar{k}}\right) .
\end{aligned}
$$

We generate the ensemble of pseudoparticles with random color orientations $O_{k}$ and random centers $z_{k}$. The instanton sizes $\rho_{k}$ are randomly taken according to Eq.(2). In spite of simple sum ansatz for multi-instanton system, the interaction among these pseudoparticles would be included effectively in the instanton size distribution.

Above procedures are performed in the continuum theory. Here, we introduce a lattice on this gauge configuration and define the link variables, $U_{\mu}(s)=\exp \left(i a A_{\mu}(s)\right)$. The string tension is estimated from the $\mathrm{SU}(2)$ Wilson loop,

$W[C] \equiv \operatorname{tr} P \exp \left[i \oint_{C} A_{\mu} d x_{\mu}\right]=\operatorname{tr} \prod_{C} U_{\mu}(s)$,

also in the multi-instanton system. In order to smooth out the ultra-violet noise, we will use the smeared link variable,

$\tilde{U}_{\mu} \equiv \frac{U_{\mu}+\sum_{\nu \neq \pm \mu} U_{\nu}(s) U_{\mu}(s+\hat{\nu}) U_{\nu}^{\dagger}(s+\hat{\mu})}{\left\|U_{\mu}+\sum_{\nu \neq \pm \mu} U_{\nu}(s) U_{\mu}(s+\hat{\nu}) U_{\nu}^{\dagger}(s+\hat{\mu})\right\|}$

instead of $U_{\mu}$ in the actual calculation, although such replacement never changes the string tension expect for reduction of the error bar.

\section{Results and Concluding Remarks}

We take the standard instanton density $(N / V)^{\frac{1}{4}}=1 \mathrm{fm}^{-1}=200 \mathrm{MeV}$,2,3, with the equal numbers of instantons and anti-instantons; $N_{I}=N_{\bar{I}}=N / 2$. We adopt the lattice with the total volume $V=(3.0 \mathrm{fm})^{4}$, and take the different lattice spacing $a=0.150 \mathrm{fm}$ and $a=0.125 \mathrm{fm}$ on the $20^{4}$ and $24^{4}$ lattices, respectively.

Fig.1 shows the Wilson loop behavior in the multi-instanton system. In the long-range region, the Wilson loop obeys the area law, which indicates existence of the linear confining potential. As shown in Fig.2, the static quark potential $V(R)$ increases in the infrared region, and is approximately proportional to the inter-quark distance $R$. It is remarkable that the string tension is obtained as $\sigma \simeq 1 \mathrm{GeV} / \mathrm{fm}$ in this multi-instanton system, which agrees with the standard value.

By comparing the two different lattice spacing cases, above results are expected to hold in continuum limit. Further analyses show that small instantons do not contribute the linear confining potential and large instantons play an essential role on the long-range physics. 


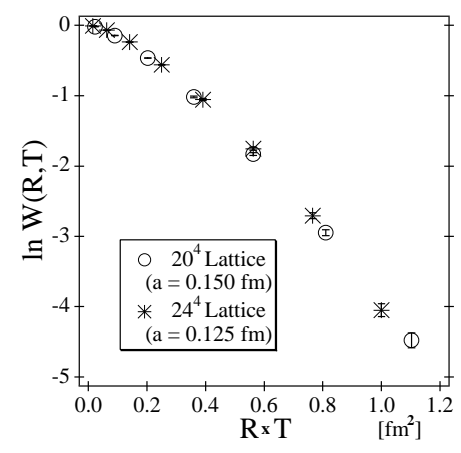

Fig.1: The Wilson loop $\ln W[R, T]$ vs. area $R \times T$ in the multi-instanton system.

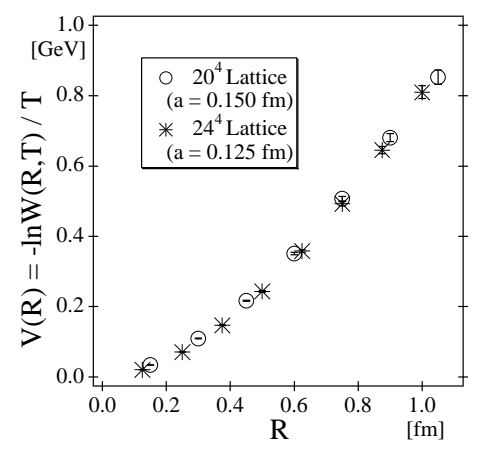

Fig.2: The static quark potential in the multi-instanton system.

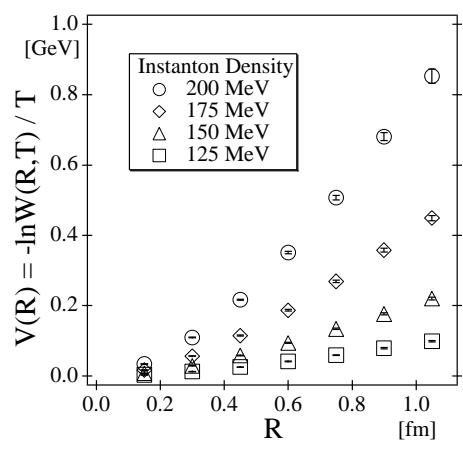

Fig.3: The static quark potential for various instanton densities.
Finally, Fig.3 shows the static potential behaviors for various instanton densities. We take the four cases with $(N / V)^{\frac{1}{4}}=125,150,175$ and $200 \mathrm{MeV}$, and use the $20^{4}$ lattice with $a=$ $0.150 \mathrm{fm}$. As the instanton density becomes lower, the slope of the potential tends to decrease monotonously. This tendency indicates that the string tension depends on the instanton density directly. Therefore, the disappearance of instantons near the critical temperature would be important for the deconfinement phase transition.

In conclusion, the main feature of the nonperturbative QCD vacuum would be characterized by instantons and anti-instantons, which cause the color randomness and provide monopole condensation [15,16]. We have studied that the confinement properties in the multi-instanton system by using the numerical analyses of the Wilson loop. In the infrared region, the static quark potential is found to be proportional to the interquark distance, $V(R) \simeq \sigma R$. For the standard instanton density $(N / V)^{\frac{1}{4}}=200 \mathrm{MeV}$, we have obtained $\sigma \simeq 1 \mathrm{GeV} / \mathrm{fm}$ in the multi-instanton system. Our numerical study suggests that instantons play an substantial role not only monopole condensation but also the confinement properties directly.

\section{REFERENCES}

1. A. Belavin, A. Polyakov, A. Shvarts and Yu. Tyupkin, Phys. Lett. 59B (1975) 85.
2. D. Diakonov and V. Petrov, in Nonperturbative Approaches to QCD (PNPI,1995) 239.

3. For a recent review see T. Schäfer and E. Shuryak, hep-ph/9610451.

4. G. 't Hooft, Nucl. Phys. B190 (1981) 455.

5. H. Suganuma, S. Sasaki and H. Toki, Nucl. Phys. B435 (1995) 207.

6. O. Miyamura, Phys. Lett. B353 (1995) 91; Nucl. Phys. B(Proc.Suppl.)42 (1995) 538.

7. O. Miyamura and S. Origuchi in Color Confinement and Hadrons (World Scientific, 1995) 235.

8. H. Suganuma, H. Ichie, S. Sasaki and H. Toki, in Color Confinement and Hadrons (1995) 65.

9. S. Thurner, H. Markum and W. Sakuler, in Color Confinement and Hadrons (1995) 77.

10. H. Suganuma, A. Tanaka, S. Sasaki and O. Miyamura, Nucl. Phys. B(Proc.Suppl.) 47 (1996) 302.

11. M. Chernodub and F. Gubarev, JETP Lett. 62 (1995) 100.

12. A. Hart and M. Teper, Phys. Lett. B371 (1996) 261.

13. R. Brower, K. Originos and C. Tan, Phys. Rev. D55 (1997) 6313.

14. H. Suganuma, S. Sasaki, H. Ichie, H. Toki and F. Araki, in Frontier '96 (World Scientific) 177 .

15. M. Fukushima, A. Tanaka, S. Sasaki, H. Suganuma, H. Toki and D. Diakonov, Nucl. Phys. B (Proc.Suppl.) 53 (1997) 494. 16. M. Fukushima, S. Sasaki, H. Suganuma, 
A. Tanaka, H. Toki and D. Diakonov, Phys. Lett. B399 (1997) 141. 